\title{
AVALIAÇÃO DA INFLUÊNCIA DA MATÉRIA ORGÂNICA NO DESEMPENHO DO PROCESSO DE DESAMONIFICAÇÃO
}

\author{
M. CORADI ${ }^{1}$, H. M. SOARES ${ }^{1}$ e A. KUNZ ${ }^{2}$ \\ ${ }^{1}$ Universidade Federal de Santa Catarina, Departamento de Engenharia Química e Engenharia \\ de Alimentos \\ 2 Embrapa Suínos e Aves \\ E-mail para contato: michaela_coradi@hotmail.com
}

\begin{abstract}
RESUMO - Um dos maiores desafios da atividade suinícola está associado ao tratamento do efluente gerado e a possibilidade do reuso da água tratada na cadeia produtiva. Processos convencionais de tratamento, como biodigestores, não removem toda matéria orgânica do efluente, tampouco os seus nutrientes. Novos processos, como o de desamonificação, com melhores custos-benefícios, surgem como alternativas promissoras para a remoção de compostos nitrogenados. No presente trabalho, avaliou-se a influência da concentração de matéria orgânica na eficiência do processo de desamonificação. Para tanto, inoculou-se um reator de leito móvel com fluxo ascendente, $30 \%$ v/v de meio suporte, operado com aeração e alimentação intermitente, a $25^{\circ} \mathrm{C}$. Os resultados mostraram que, na presença de matéria orgânica, houve uma competição entre BOA e bactérias heterotróficas por $\mathrm{OD}$, comprometendo a estabilidade do processo quando a relação $\mathrm{C} / \mathrm{N}$ foi superior a 2,0 mgCOT.(mgN-NH$\left.{ }_{4}^{+}\right)^{-1}$, nas condições operacionais estudadas.
\end{abstract}

\section{INTRODUÇÃO}

Nos últimos anos, o Brasil tem se destacado na produção e exportação de carne suína, devido, principalmente a fatores como a grande extensão territorial e a oferta de matéria prima para a produção (Abipecs, 2014). Esta grande produtividade gera uma quantidade considerável de resíduos, com potencial poluidor elevado. Em função disto, sistemas de tratamento vêm sendo estudados para reduzir as emissões de compostos orgânicos voláteis, controlar a proliferação de odores, a mineralização de nutrientes, objetivando a melhora das propriedades fertilizantes destes resíduos gerados, bem como a recuperação de energia através da produção de biogás (Figueroa et al., 2012) a fim de atender as legislações vigentes e sobretudo reduzir impactos ambientais causados pela atividade.

Segundo Kunz et al. (2005), a tecnologia de digestão anaeróbia por biodigestores para estabilização e remoção de matéria orgânica de dejetos suínos é largamente utilizada. $\mathrm{O}$ inconveniente deste processo é que os compostos nitrogenados contidos em abundância nos dejetos, tais como proteínas, aminoácidos ou ureia, são convertidos principalmente em amônio, o qual não é degradado em condições anaeróbias (Figueroa et al., 2012). Diante disto, torna-se necessário encontrar soluções sustentáveis para a remoção de nitrogênio de efluentes suinícolas antes de serem lançados em corpos d'água ou serem reutilizados na agricultura ou no próprio processo produtivo. 
Por apresentar capacidade de remoção de altas concentrações de nitrogênio, o processo ANAMMOX vem sendo estudado como alternativa para a remoção das formas nitrogenadas presentes no efluente suinícola. (Molinuevo et al., 2009; Casagrande et al. 2011). Para aplicar o processo ANAMMOX, é necessária uma etapa de nitrificação parcial, onde aproximadamente metade do amônio contido no efluente é convertido a nitrito, proporcionando as concentrações exigidas pela estequiometria do processo (Paredes et al., 2007). Este sistema pode ser obtido utilizando dois reatores diferentes, um para o processo de nitrificação parcial e outro para o processo ANAMMOX (Van Dongen et al., 2001, Fux et al., 2002) ou em um sistema de reator único onde uma cultura mista de bactérias anammox e bactérias oxidadoras de amônia (BOA) convivem simultaneamente (Third et al., 2001; Cho et al., 2011).

O sistema de reator único, conhecido por processo de desamonificação, torna-se competitivo aos demais processos em relação ao seu custo de investimento, como construção, manutenção, economia de energia, uma vez que consome menos oxigênio que processos convencionais de remoção de nitrogênio (Third et al., 2001) e pode operar a temperatura ambiente (Vázquez-Padín et al., 2009).

Este conjunto de fatores abre a possibilidade de aplicar este processo no tratamento de dejetos suínos como sendo, por exemplo, um pós-tratamento de lagoas anaeróbias ou biodigestores. Em contraste, estes efluentes, mesmo após passar por estes pré-tratamentos, ainda contêm concentrações consideráveis de matéria orgânica dissolvida, que podem interferir na eficiência do processo de desamonificação (Zhang et al., 2012).

Considerando estes aspectos, o presente trabalho teve como objetivo avaliar a influência da matéria orgânica na eficiência da remoção de nitrogênio pelo processo de desamonificação, combinando nitrificação parcial e ANAMMOX em um reator único, à temperatura de $25^{\circ} \mathrm{C}$.

\section{MATERIAL E MÉTODOS}

\subsection{Descrição do reator e condições operacionais}

O sistema era composto por um reator de leito móvel, com fluxo ascendente, construído a partir de um cone Imhoff de acrílico, com volume útil de 1,2 L, um banho termostatizado e um sedimentador para reciclo de biomassa, também feito a partir de cone Imhoff, com volume útil de $1 \mathrm{~L}$, operando com alimentação e aeração intermitente, com ciclos de 30 minutos, sendo 8 minutos aerando e alimentando e 22 minutos em fase anóxica sem alimentação, conforme ilustrado na Figura 1. Utilizou-se 30\% v/v de meio suporte do tipo Kaldnes com a finalidade de formar biofilme e proteger a biomassa da tensão de cisalhamento causada pela fluidização durante a aeração e reduzir a perda de biomassa do reator. A vazão de ar foi regulada de forma a manter o OD do meio menor que $0,5 \mathrm{mg} . \mathrm{L}^{-1}$ e variou de $8,3 \times 10^{-3} \mathrm{~L}$. $\mathrm{L}^{-}$ ${ }^{1} \cdot \mathrm{min}^{-1}$ a $83,3 \times 10^{-3} \mathrm{~L} \cdot \mathrm{L}^{-1} \cdot \mathrm{min}^{-1}$.

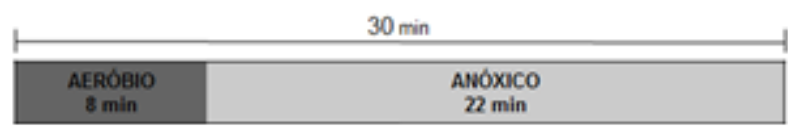

Figura 1 - Ciclo de aeração e alimentação do Reator. 
O reator foi inoculado com 1,4 gSSV.L ${ }^{-1}$ de biomassa anammox e 0,10 gSSV.L ${ }^{-1}$ de nitrificante, definidas através de ensaios cinéticos preliminares de suas respectivas velocidades específicas de consumo de nitrogênio. A temperatura foi controlada em $25^{\circ} \mathrm{C} \pm 3$. $\mathrm{O}$ pH não foi ajustado e se manteve entre 7,67 e 8,24. A alimentação foi realizada com meio de cultura sintético, com concentração de nitrogênio amoniacal, na forma de $\mathrm{NH}_{4} \mathrm{Cl}$, de 200 $\mathrm{mgN}-\mathrm{NH}_{4}+\mathrm{L}^{-1}$ e carga de $0,4 \mathrm{gN}-\mathrm{NH}_{4}{ }^{+} \cdot \mathrm{L}^{-1} \cdot \mathrm{d}^{-1}$, adaptado de Magrí et al. (2012), com a seguinte composição: $\mathrm{NH}_{4} \mathrm{Cl}\left(763,8 \mathrm{mg} . \mathrm{L}^{-1}\right), \mathrm{K}_{2} \mathrm{HPO}_{4}\left(100 \mathrm{mg} \cdot \mathrm{L}^{-1}\right), \mathrm{NaHCO}_{3}\left(1941 \mathrm{mg} . \mathrm{L}^{-1}\right)$, $\mathrm{Na}_{2} \mathrm{CO}_{3}\left(260,7 \mathrm{mg} . \mathrm{L}^{-1}\right), \mathrm{MgSO}_{4} .7 \mathrm{H}_{2} \mathrm{O}\left(60 \mathrm{mg} . \mathrm{L}^{-1}\right), \mathrm{FeSO}_{4} \cdot 7 \mathrm{H}_{2} \mathrm{O}\left(8 \mathrm{mg} \cdot \mathrm{L}^{-1}\right), \mathrm{CaCl}_{2} \cdot 2 \mathrm{H}_{2} \mathrm{O}(8$ mg. $\left.\mathrm{L}^{-1}\right)$ e $1 \mathrm{~mL} . \mathrm{L}^{-1}$ da solução de elementos traço: $\mathrm{ZnSO}_{4}\left(1247 \mathrm{mg} . \mathrm{L}^{-1}\right), \mathrm{MnSO}_{4}\left(1149 \mathrm{mg} . \mathrm{L}^{-}\right.$ $\left.{ }^{1}\right), \mathrm{CuSO}_{4} \cdot 5 \mathrm{H}_{2} \mathrm{O}\left(44 \mathrm{mg} \cdot \mathrm{L}^{-1}\right), \mathrm{Al}_{2}\left(\mathrm{SO}_{4}\right)_{3} \cdot 14 \mathrm{H}_{2} \mathrm{O}\left(201 \mathrm{mg} \cdot \mathrm{L}^{-1}\right), \mathrm{Na}_{2} \mathrm{MoO}_{4} \cdot 2 \mathrm{H}_{2} \mathrm{O}\left(129 \mathrm{mg} . \mathrm{L}^{-1}\right)$, $\mathrm{CoCl}_{2} \cdot 6 \mathrm{H}_{2} \mathrm{O}\left(30 \mathrm{mg} \cdot \mathrm{L}^{-1}\right), \mathrm{KCl}\left(100 \mathrm{mg} \cdot \mathrm{L}^{-1}\right)$ e EDTA $\left(975 \mathrm{mg} \cdot \mathrm{L}^{-1}\right)$.

\subsection{Avaliação da influência da matéria orgânica}

Os testes foram realizados em batelada, dentro do Reator. Utilizou-se o mesmo efluente sintético da alimentação, com adição de acetado de sódio como fonte de carbono orgânico. A vazão de ar durante os testes foi a mesma que o processo estava operando, $35 \mathrm{~mL} \cdot \mathrm{min}^{-1}$. A temperatura do processo foi mantida constante, em $25^{\circ} \mathrm{C}$.

Testaram-se as seguintes relações $\mathrm{C} / \mathrm{N}$ : 0 mgCOT.mgN-1, 0,3 mgCOT.mgN-1, 2 mgCOT.mgN-1, 3,5 mgCOT.mgN-1, 4,5 mgCOT.mgN-1 e 5 mgCOT.mgN-1. Para cada relação $\mathrm{C} / \mathrm{N}$ testada, manteve-se constante a concentração inicial de amônio, na forma de $\mathrm{NH}_{4} \mathrm{Cl}$, em $200 \mathrm{mgN}-\mathrm{NH}_{4}{ }^{+}$. $\mathrm{L}^{-1}$, variando-se apenas a concentração de COT. Os testes tiveram duração de 8 horas. Coletou-se $5 \mathrm{~mL}$ de amostra a cada 30 minutos.

\subsection{Métodos analíticos}

A análise de $\mathrm{N}_{-} \mathrm{NH}_{4}{ }^{+}$foi realizada pelo método colorimétrico de Nessler (Vogel, 1981), $\mathrm{N}-\mathrm{NO}_{2}{ }^{-}$e $\mathrm{N}_{-\mathrm{NO}_{3}}{ }^{-}$por método colorimétrico, alcalinidade total por titulação e COT, com o princípio da queima a $950^{\circ} \mathrm{C}$ e medido $\mathrm{CO}_{2}$ por detector NDIR por infravermelho, conforme Apha (2012).

\section{RESULTADOS E DISCUSSÃO}

As Figuras 2 e 3 apresentam o comportamento da velocidade de consumo de amônio em função do aumento da relação $\mathrm{C} / \mathrm{N}$ e da eficiência do processo em função do aumento do COT, respectivamente, considerando que, devido a curta duração dos ensaios e o lento crescimento da biomassa, a concentração celular permaneceu constante.

Como pode-se visualizar na Figura 2, as velocidades de consumo de amônio, diminuíram com o aumento da relação $\mathrm{C} / \mathrm{N}$, indicando que a atividade do processo de desamonificação reduziu com o aumento do COT, o que se confirma através da Figura 3, a qual apresenta uma queda de eficiência na remoção de $\mathrm{N}_{-} \mathrm{NH}_{4}{ }^{+}$com o aumento da concentração de COT.

Durante a realização dos ensaios, houve consumo de carbono orgânico, sugerindo a presença de bactérias heterotróficas aeróbias e desnitrificantes. 


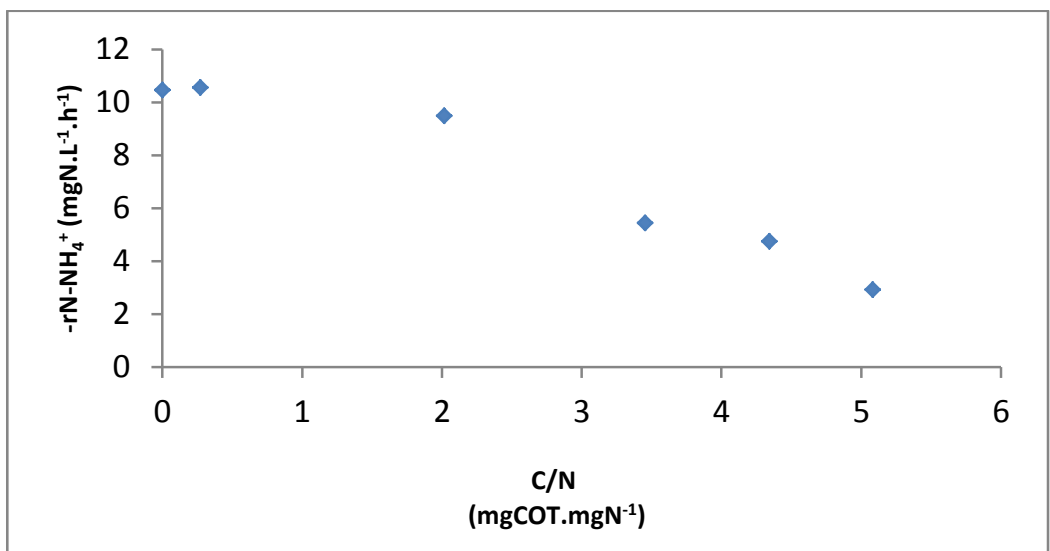

Figura 2 - Comportamento da velocidade de consumo de $\mathrm{N}_{-} \mathrm{NH}_{4}{ }^{+}$em função do aumento da razão $\mathrm{C} / \mathrm{N}$.

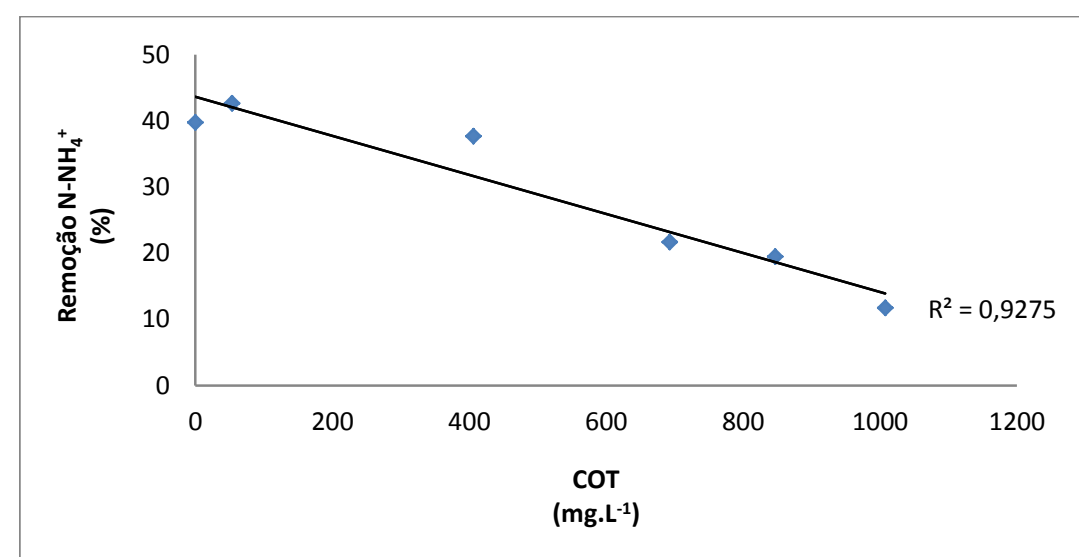

Figura 3 - Eficiência de remoção de $\mathrm{N}^{-\mathrm{NH}_{4}}{ }^{+}$em função do aumento da concentração de COT.

Segundo Rittmann e McCarty (2001), quando microrganismos usam substrato como doador de elétrons para síntese, uma porção desses elétrons $\left(\mathrm{f}_{\mathrm{e}}{ }^{\circ}\right)$ é normalmente transferida para o aceptor de elétrons para proporcionar energia, para então converter a outra porção de elétrons $\left(\mathrm{f}_{\mathrm{s}}^{\circ}\right)$, em células microbianas. Ainda de acordo com os autores, as bactérias nitrificantes têm muito menos elétrons convertidos em células $\left(\mathrm{f}_{\mathrm{s}}^{\circ}=0,1\right)$ comparadas a bactérias heterotróficas aeróbias $\left(\mathrm{f}_{\mathrm{s}}^{\circ}=0,6-0,7\right)$, logo, o crescimento heterotrófico é muito maior que o das bactérias nitrificantes. Os autores também afirmam que as bactérias desnitrificantes heterotróficas, por serem facultativas, têm características cinéticas similares às bactérias heterotróficas aeróbias. Portanto, bactérias desnitrificantes e heterotróficas aeróbias são muito mais favorecidas que as bactérias nitrificantes na competição por substrato, no caso, o oxigênio dissolvido.

A redução da velocidade de consumo de amônio do processo com o aumento do carbono orgânico disponível justifica-se devido que, uma maior concentração de COT, aumenta o consumo de oxigênio por bactérias heterotróficas aeróbias. Essa competição por OD, suprime a atividade das BOA. 
Tendo menos oxigênio disponível, a conversão de nitrogênio amoniacal em nitrito é consequentemente reduzida. Sem nitrito em condição estequiométrica suficiente, a capacidade das bactérias anammox também acaba sendo suprimida e, por estas serem mais sensíveis, podem ter sido desfavorecidas, comprometendo o funcionamento do processo. A afinidade entre nitrito e as bactérias anammox é muito maior que entre nitrito e bactérias desnitrificantes (Ahn, 2006), porém, por estarem em condições que as desfavorecem, como a exposição ao OD e a matéria orgânica, esta afinidade das bactérias anammox pelo nitrito pode ter sido reduzida e, por consequência, podem ter sido suprimidas pelo processo de desnitrificação uma vez que, com o aumento do COT disponível, o nitrito produzido pelas BOA possa ter desnitrificado.

A Figura 4 relaciona o consumo de $\mathrm{COT}$ e $\mathrm{N}_{-} \mathrm{NH}_{4}{ }^{+}$em função do aumento da relação $\mathrm{C} / \mathrm{N}$.

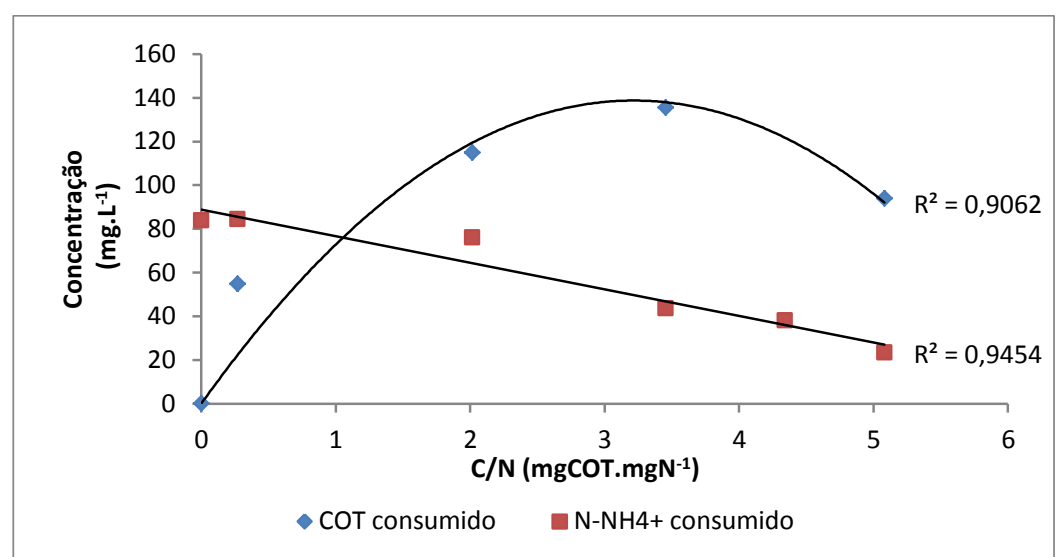

Figura 4 - Relação entre o consumo de $\mathrm{COT}$ e $\mathrm{N}^{-\mathrm{NH}_{4}}{ }^{+}$em função do aumento da relação $\mathrm{C} / \mathrm{N}$.

O comportamento do processo, representado no gráfico da Figura 4, sugere que a partir da relação $\mathrm{C} / \mathrm{N}$ de $3,5 \mathrm{mgCOT} . \mathrm{mgN}^{-1}$ o consumo de COT por bactérias heterotróficas diminuiu, porém, devido a dispersão dos pontos experimentais para as últimas relações $\mathrm{C} / \mathrm{N}$ testadas, nas análises de COT, não é possível afirmar que o patamar do gráfico tenha de fato este comportamento, podendo este ser constante e as bactérias heterotróficas terem atingido a saturação.

Através da Figura 4 também é possível observar que o consumo de nitrogênio amoniacal continuou diminuindo, mesmo com essa saturação do consumo de COT, indicando que, além de ter sido suprimido pela falta de OD disponível, o processo pode ter sido inibido pela presença de matéria orgânica. Esta inibição das bactérias anammox pela presença de altas concentrações de matéria orgânica é relatada na literatura. Dapena-Mora et al.(2007) avaliaram a inibição da atividade específica anammox utilizando acetato de sódio como fonte de matéria orgânica e encontraram que concentrações de COT de 600 e 1200 mgCOT.L ${ }^{-1}$ resultaram em uma inibição de 22 e $70 \%$, respectivamente.

Em relação ao processo, estudos diferem quanto a quantidade de COT e da relação C/N a ser aplicada, em função da configuração do reator, suas condições operacionais e características do efluente. 
Estudos realizados por Zhang et al. (2012) avaliaram a influência de diferentes relações $\mathrm{C} / \mathrm{N}$ para efluente suinícola pós-biodigestor na eficiência de remoção de nitrogênio pelo processo de desamonificação em um reator de biofilme do tipo RBS em escala de bancada, com volume útil de $6,5 \mathrm{~L}$, operado a $30^{\circ} \mathrm{C}$ e condições controladas de OD. Cloreto de amônio e acetato foram utilizados para as correções das razões $\mathrm{C} / \mathrm{N}$ do efluente. Neste estudo, a concentração de OD precisou ser aumentada com a adição de matéria orgânica para manter a estabilidade do processo. $\mathrm{O}$ aumento da relação $\mathrm{C} / \mathrm{N}$ para 1,24 causou uma queda na eficiência de remoção de nitrogênio. A relação $\mathrm{C} / \mathrm{N}$ ideal para as condições operacionais neste estudo foi 0,65. Outros estudos, realizados por Winkler et al. (2012), avaliaram a remoção de nitrogênio e DQO pelo processo de desamonificação em um reator granular, operado em condições aeróbia e anóxica, a temperatura ambiente, encontrando estabilidade do processo até a relação $\mathrm{C} / \mathrm{N}$ de 0,5 . Chen et al. (2013) estudaram a aplicação do processo no tratamento de efluente proveniente da criação de tartarugas e obtiveram um eficiência de remoção de nitrogênio total e de DQO de $85 \%$ e $56 \%$, respectivamente, trabalhando uma relação C/N de aproximadamente 2. Figueroa et al. (2012) não observaram efeito significativo no processo trabalhando-se nas relações $\mathrm{DQO} / \mathrm{N}$ entre 2,4 e $5 \mathrm{gDQO} / \mathrm{gN}$ e atribuem isto a diferença na biodegradabilidade dos diferentes efluentes ao passarem por pré-tratamentos.

Neste estudo, não se observou diferença significava na eficiência de remoção de nitrogênio amoniacal do processo trabalhando-se com relações $\mathrm{C} / \mathrm{N}$ abaixo de 2 mgCOT.mgN-NH${ }_{4}^{+}$, para as condições operacionais estudas.

\section{CONCLUSÃO}

Os testes avaliando a influência da presença de matéria orgânica no processo de desamonificação mostraram a ocorrência de uma competição entre BOA e bactérias heterotróficas por OD, o qual se tornou o limitante do processo. Essa competição entre BOA e heterotróficas reduziu a conversão de nitrogênio amoniacal a nitrito, suprimindo também o processo ANAMMOX. Nas condições estudadas, relações $\mathrm{C} / \mathrm{N}$ maiores que 2,0 mgCOT.mgN ${ }^{-1}$ desestabilizaram e descaracterizaram o processo de desamonificação.

\section{REFERÊNCIAS}

ABIPECS (Associação Brasileira da Indústria Produtora e Exportadora de Carne Suína). Produção Mundial de Carne Suína. Disponível em: <http://www.abipecs.org.br/pt/estatisticas/mundial/exportacao.html>. Acesso em: 16/01/2014.

AHN, Y.H. Sustainable nitrogen elimination biotechnologies: A review. Process Biochemistry, v. 41, p.1709-1721, 2006.

APHA - American Public Health Association. Standard methods for the examination of water and wastewater. 22 ed. Washington, DC: American Public Health Association, 2012.

CASAGRANDE, C.G.; KUNZ, A.; SOARES, H.M., DE PRÁ, M.C.; SCHIERHOLT NETO, G.F. Establishment of anammox process in sludge samples collected from swine wastewater treatment system. Engenharia Agrícola, v. 31, n. 6, 2011. 
CHEN, C.; HUANG, X.; LEI, C.; ZHANG, T.C.; WU, W. Effect of Organic Matter Strength on Anammox for Modified Greenhouse Turtle Breeding Wastewater Treatment. Bioresource Technology, v. 148, p. 172-179, 2013.

CHO, S.; FUJII, N.; LEE, T.; OKABE, S. Development of a simultaneous partial nitrification and anaerobic ammonia oxidation process in a single reactor. Bioresource Technology, v. 102, p. $652-659,2011$.

DAPENA-MORA, A.; FERNÁNDEZ, I.; CAMPOS, J.L.; MOSQUERA-CORRAL, A.; MÉNDEZ, R.; JETTEN, M.S.M. Evaluation of activity and inhibition effects on Anammox process by batch tests based on the nitrogen gas production. Enzyme and Microbial Technology, v. 40, p. 859-865, 2007.

FIGUEROA, M.; VÁZQUEZ-PADÍN, J.R.; MOSQUERA-CORRAL, A.; CAMPOS, J.L. Is the CANON reactor an alternative for nitrogen removal from pre-treated swine slurry? Biochemical Engineering Journal, v. 65, p. 23-29, 2012.

FUX, C.; BOEHLER, M.; HUBER, P.; BRUNNER, I.; SIEGRIST, H. Biological treatment of ammonium-rich wastewater by nitritation and subsequent anaerobic ammonium oxidation (anammox) in a pilot plant. Journal of Biotechnology, v. 99, p. 295-306, 2002

KUNZ, A.; HIGARASHI, M. M.; OLIVEIRA, P. O. Tecnologias de manejo e tratamento de dejetos de suínos estudadas no Brasil. Cadernos de Ciência \& Tecnologia, v.22, p.651-665, 2005 .

MAGRÍ, A., VANOTTI, M. B., SZÖGI, A. A., CANTRELL, K. B. Partial Nitritation of Swine Wastewater in View of its Coupling with the Anammox Process. Journal of Environmental Quality, v. 41, p. 1989-2000, 2012.

MOLINUEVO, B.; GARCÍA, M.C.; KARAKASHEV, D.; ANGELIDAKI, I. Anammox for ammonia removal from pig manure effluents: Effect of organic matter content on process performance. Bioresource Technology, v. 100, p. 2171-2175, 2009.

PAREDES, D.; KUSCHK, P.; MBWETTE, T.S.A.; STANGE, F.; MÜLLER, R.A.; KÖSER, H. New Aspects of Microbial Nitrogen Transformations in the Context of Wastwater Treatment - A Review. Engineering in Life Sciences, v.7, n. 1, p. 13-15, 2007.

RITTMANN, B. E.; MCCARTY, P. L. Environmental biotechnology: principles and applications. New York: McGraw Hill Book Co, 2001.

THIRD, K.A.; SLIEKERS, A.O.; KUENEN, J.G.; JETTEN, M.S.M. The CANON System (Completely Autotrophic Nitrogen-removal Over Nitrite) under Ammonium Limitation: Interaction and Competition between Three Groups of Bacteria. Systematic and Applied Microbiology, v. 24, p. 588-596, 2001. 
VAN DONGEN, L. G. J. M.; JETTEN, M. S. M.; VAN LOOSDRECHT, M. C. M. The Combined SHARON/ANAMMOX Process. Stowa: Foundation for Applied Water Research. IWA Publishing. London, 2001.

VÁZQUEZ-PADÍN, J.R.; POZO, M.J.; JARPA, M.; FIGUEROA, M.; FRANCO, A.; MOSQUERA-CORRAL, A.; CAMPOS, J.L.; MÉNDEZ, R. Treatment of anaerobic sludge digester effluents by the CANON process in an air pulsing SBR. Journal os Hazardous Materials, v. 166, p. 336-341, 2009.

VOGEL, A. I. Análise Inorgânica Quantitativa. 4.a ed. Rio de Janeiro: Guanabara, 1981.

WINKLER, M.K.H.; KLEEREBEZEM, R.; VAN LOOSDRECHT, M.C.M. Integration the anammox into the aerobic granular sludge process for main stream wastewater treatment at ambient temperature. Water Research, v. 46., p. 136-144, 2012.

ZHANG, Z. LI, Y.; CHEN, S.; WANG, S.; BAO, X. Simultaneous nitrogen and carbon removal from swine digester liquor by Canon process and denitrification. Bioresource Technology, v. 114, p. 84-89, 2012. 